\title{
Modeling and investigating the stability of a solution to the inverse problem of signal separation
}

\author{
V.A. Zasov ${ }^{1}$, Ye.N. Nikonorov ${ }^{1}$

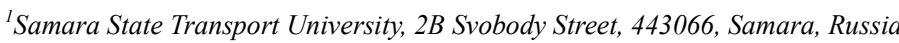

\begin{abstract}
This paper proposes a method for modeling and analyzing the stability of a solution to the inverse problem of extracting individual signals from an additive mixture of several signals that come to measurement points from various signal sources inaccessible for direct measurement. Stability analysis is accomplished by determining those intervals (singular intervals) for parameters of a signal formation model in which steady signal separation is achievable. We have developed algorithms to calculate singular intervals for different parameter variations of a signal formation model - absolute, relative, critical, and their combinations - that simulate various practically significant types of model parameter perturbations that affect the stability of the solution to this inverse problem. The paper also presents the results of computer modeling for the proposed algorithms.
\end{abstract}

Keywords: signal separation; inverse problem; solution stability; signal models; singular intervals; algorithm; modeling

\section{Introduction}

Signal separation is a solution to the problem of extracting individual signals from an additive mixture of several signals that come to measurement points from various signal sources inaccessible for direct measurement. That solution is needed in many practical fields such as monitoring and diagnosis of technical facilities (e.g., vibroacoustic diagnosis), communications, medical diagnosis, and speech processing. This is because in complicated facilities, measured signals present an additive mixture of signals received from many components, and in most practical applications the extraction of parameters that describe the state of specific components is impossible without signal separation.

In addition, signal separation enables further parallel processing of each extracted signal, thereby improving the efficiency of data-processing systems.

Problem of signal separation relates to the class of inverse problems, which may be ill-posed in the general case. From that, it follows that the solution may be unsteady because slight changes in the parameters of the mixing matrix $\mathbf{H}$ of the signal formation model or in characteristics of source signals lead to impermissibly large changes in the solution: an unstable computation of source signals [1,2]. For a stable solution, the parameters of the object described by the signal formation model must satisfy several prior restrictions [3]. For instance, the mixing matrix must be invertible; the polynomials describing the transfer functions of channels must not have common roots; the number of receivers must equal that of sources.

In practice, prior restrictions may be violated since object parameters may change because of the object's evolution in time, measurement error, fabrication inaccuracies, and other causes that often are unpredictable. Thus, changes in parameters mixing matrix and in characteristics of source signals may cause a steady solution to migrate toward an unstable one, unsuitable for practical use.

That is why it is important to investigate how deviations of the above source properties from those presumed a priori affect the solution's stability and to investigate deviations from requirements for channel characteristics. It is relevant that the stability of the solution to the problem of signal separation should be determined a priori by calculating whether the parameters of the signal formation model fall within the model's parameter intervals in which stability is achievable. Currently the methods described below are used to analyze the stability of the solution to the inverse problem of signal separation.

Given that the signal formation model in the frequency domain is described, for each frequency, by a system of linear algebraic equations[ ], the stability of signal separation is determined by the stability of the solution to equation systems, which, as is known (see, e.g., ref. [4]), is determined by the condition number cond $(\mathbf{H})$. Thus, by calculating the condition number, one can evaluate the stability of signal separation: The higher the condition number, the worse the stability.

As reference [5] shows, for the matrix of parameter intervals, the minimal norm reducing the initial matrix $\mathbf{H}$ to the degenerated matrix $\operatorname{det}(\mathbf{H}+\Delta \mathbf{H})=0$ equals $\|\Delta \mathbf{H}\|_{2}=1 /\left\|\mathbf{H}^{-1}\right\|_{2}=\sigma_{M}$. With the value (real number) $\|\Delta \mathbf{H}\|_{2}$, one can also evaluate the stability of the solution to the problem of signal separation.

Stability analysis methods based on using condition numbers cond $(\mathbf{H})$ and matrix norm $\|\Delta \mathbf{H}\|_{2}$ exhibit limited functionality. Indeed, values cond $(\mathbf{H})$ and $\|\Delta \mathbf{H}\|_{2}$ are integral estimates of stability, and they do not allow singular intervals $\Delta H$ to be determined in singular-interval matrices $\Delta \mathbf{H}$, which is important for practical applications. It is evident that the knowledge, in matrices, of elements (singular intervals) close to zero makes it possible to determine elements of mixing matrices $\mathbf{H}$ on which the stability of the problem of signal separation mainly depends. In other words, values cond $(\mathbf{H})$ and $\|\Delta \mathbf{H}\|_{2}$ do not take into account the structure of a perturbation (for the same condition numbers and matrix norms, there can be an infinite number of perturbation realizations). But in practice, perturbations can have a structure: Each matrix element can have its own perturbation that is unlike the others, and that perturbation can, in turn, be absolute, relative, or critical — or a combination of the three.

Reference [6] proposes a method for analyzing the stability of the solution to a system of linear algebraic equations, offering 
broader functionality compared with the methods described above. The method can be used for analyzing and verifying the stability of the solution to the problem of signal separation. The algorithm that uses that method determines the direction of the worst parameter variations that cause instability (singularity). If, in a set parameter interval, the condition number for absolute or relative variations has increased significantly (e.g., exceeded a threshold), a solution within this interval is assumed unstable. Thus, the algorithm makes it possible to analyze the stability of a solution for signal formation models at a set variation value of mixing-matrix elements.

The method proposed in [10] does not solve the problem of determining the matrix $\Delta \mathbf{H}$ of singular parameter intervals; nor does it enable use of complex mixing matrices $\mathbf{H}(\omega)$, and that limits the method's functionality.

Thus, our analysis of existing methods' functionality indicates the relevance of developing a method for modeling, analyzing, and verifying the stability of the solution to the problem of signal separation.

This paper proposes analyzing stability by determining singular intervals with the model's parameter variations directed toward the maximal deterioration of the solution's stability.

\section{The object of the study}

To state the problem formally, we will consider a signal formation model presented as a linear multivariable system that has $N$ inputs and $M$ outputs. The model's input signals are $s_{n}(k), n=1,2, \ldots, N$; output signals, $x_{m}(k), m=1,2, \ldots, M$. The input signals are generated by various signal sources, and the output signals may be signals of various receivers such as sensors, measurement transducers, and antennas. Let us assume that each of the $M$ outputs of the multivariable system is connected with all the $N$ inputs through linear signal transmission channels.

At any discrete instant of time $k$, the $M$-dimensional vector of sensor-measured discrete signals $\mathbf{x}(k)=\left[x_{1}(k), x_{2}(k), \ldots, x_{M}(k)\right]^{T}$ results from the $N$-dimensional vector of source signals $\mathbf{s}(k)=\left[s_{1}(k), s_{2}(k), \ldots, s_{N}(k)\right]^{T}$. The mathematical model of signal formation is described by an equation system of discrete convolution type (1), where the $m$ th observed signal is an additive mixture of channel-distorted source signals and noise [7]; that is,

$$
x_{m}(k)=\sum_{n=1}^{N} \sum_{g=0}^{G-1} h_{m n}(g, \mathbf{l}) s_{m}(k-g)+y_{m}(k),
$$

where $h_{m n}(g, \mathbf{l})$ is the element $M \times N$ of the $\mathbf{h}(g, \mathbf{l})$ matrix for the impulse characteristics of channels, and $\mathbf{y}(k)=\left[y_{1}(k), y_{2}(k), \ldots, y_{M}(k)\right]^{T}$ is the noise vector. For purposes of further discussion, we will assume that the $h_{m n}(g, \mathbf{l})$ impulse characteristics are finite and are represented by the counting number $G$. The dynamic characteristics of channels $h_{m n}(g, \mathbf{l})$ are quasistationary in that they change depending on parameter vector $\mathbf{l}$ (time, temperature, location, etc.).

In the frequency domain, model (1) is described as

$$
\mathbf{X}(\omega)=\mathbf{H}(\omega, \mathbf{l}) \cdot \mathbf{S}(\omega)+\mathbf{Y}(\omega)
$$

where $\mathbf{H}(\omega, \mathbf{l})=\left(\begin{array}{cc}H_{11}(\omega, \mathbf{l}) & H_{1 N}(\omega, \mathbf{l}) \\ H_{M 1}(\omega, \mathbf{l}) & H_{M N}(\omega, \mathbf{l})\end{array}\right)$ - is the mixing matrix $M \times N$, comprising Fourier transforms of the channels;

$\mathbf{X}(\omega)=\left[X_{1}(\omega), \quad, X_{M}(\omega)\right]^{T}$ is the vector of observed signals, and it comprises Fourier transforms of receiver signals; $\mathbf{S}(\omega)=\left[S_{1}(\omega), \quad, S_{N}(\omega)\right]^{T}$ is the vector of source signals, and it comprises Fourier transforms of source signals; $\mathbf{Y}(\omega)=\left[Y_{1}(\omega), \quad, Y_{M}(\omega)\right]^{T}$ is the noise vector, comprising Fourier transforms of noise signals. Signals of sources $\mathbf{S}(\omega)$ and of noise $\mathbf{Y}(\omega)$ are considered independent, and channels can be modeled by spectral converters such as various filters.

Generally, the solution to the problem of separating signal sources reduces to calculating the separating matrix $\mathbf{w}(g)$, which is, in terms of specific criteria, equal or close to the matrix inverse to matrix $\mathbf{h}(g, \mathbf{l})$. Thus, generally, the solution to the problem of separating source signals is the solution to system (1), and it can be expressed as

$$
s_{n}(k)=\sum_{m=1}^{M} \sum_{g=0}^{G-1} w_{n m}(g, \mathbf{l}) x_{m}(k-g),
$$

where $\mathbf{w}(g, \mathbf{l})$ is the matrix of impulse characteristics of tunable filters with $w_{n m}(g, \mathbf{l})$ elements. In the frequency domain, equation (3) can be written as

$$
\mathbf{S}(\omega)=\mathbf{W}(\omega, \mathbf{l}) \mathbf{X}(\omega)
$$

where $\mathbf{W}(\omega, \mathbf{l})=\mathbf{H}^{-1}(\omega, \mathbf{l})$. It is evident that calculating the separating matrix $\mathbf{w}(g, \mathbf{l})$ requires prior information about parameters of the signal formation model (object parameters). For separating signal sources, a variety of approaches are used that are based on various prior knowledge of the item under study. Signal separation methods can be classified into two groups-deterministic and statistical [1]. 
The deterministic group is based on prior information about characteristics of signal transmission channels - that is, on the knowledge of the matrix of impulse characteristics $\mathbf{h}(g, \mathbf{l})$, which are either measured or determined from theoretical premises.

A feature of the statistical group is that $\mathbf{h}(g, \mathbf{l})$ matrix elements are unknown explicitly, and the information used to determine input signals $\mathbf{s}(k)$ is provided by the realization of the vector of measured signals $\mathbf{x}(k)$ and the knowledge of source properties of signals $\mathbf{s}(k)$.

The deterministic group is based on principal information about signal transmission channels (statistical, frequency, amplitude, and other channel characteristics); that is, transmission channels and signals are known.

The statistical group is based on principal information about signal sources such as lacking source correlation and the knowledge of signal distribution laws. In this case, explicit information about transmission channels is unavailable, and only observed signals are known. For that reason, the methods within this group are often called "blind" [8].

Thus, the solution to the problem of separating of signal sources reduces to using a deterministic or statistical method to calculate the separating matrix $\mathbf{w}(g, \mathbf{l})$ equal or close, in terms of specific criteria, to the matrix inverse to matrix $\mathbf{h}(g, \mathbf{l})$.

There are separation methods that fall within neither group because they use information both about channels and the properties of signal sources (e.g., adaptive noise concellation [9]).

From general solution (3) it follows that the problem of signal separation relates to the class of inverse problems, which may be ill-posed in the general case.

Our paper aims to:

-Develop an algorithm for modeling the problem of signal separation with a solution whose stability is variable by setting variations for the parameters of the signal formation model.

-Develop algorithms for analyzing and veryfying stability by determining parameter intervals in which stable signal separation is achievable for various practically significant variations of model parameters.

This paper investigates the stability of the solution to the problem of signal separation with varied parameters of channels $H_{m n}(\omega, \mathbf{l})$, constituting the mixing matrix $\mathbf{H}(\omega, \mathbf{l})$.

\section{Methods. Algorithms for Modeling, Analyzing, and Verifying the Stability of the Solution to the Problem of Signal Separation}

\subsection{Mathematical Signal Formation Model with the Capability to Set Variations for Channel Parameters}

To investigate how prior indefinite perturbations affect stability, we propose introducing singular-variation blocks for parameters of channels $\delta h_{m n}$ into the signal formation model. Then the signal formation model with parameter variations shown in figure 1 will take the form of [7]

$$
x_{m}(k)=\sum_{n=1}^{N} \sum_{g=0}^{G-1}\left(h_{m n}(g, \mathbf{l})+\delta h_{m n}(g, \mathbf{l})\right) s_{m}(k-g)+y_{m}(k)
$$

In the frequency domain, expression (5) can be written as

$$
\mathbf{X}(\omega)=(\mathbf{H}(\omega, \mathbf{l})+\delta \mathbf{H}(\omega, \mathbf{l})) \cdot \mathbf{S}(\omega)+\mathbf{Y}(\omega),
$$

where $\delta \mathbf{H}(\omega, \mathbf{l})$ is the matrix of singular parameter variations.

Unlike objectively existing perturbations, parameter variations in the model for stability studies can be modeled by introducing a block for setting types of variation. Thus, mathematical model (5) can be used to investigate how perturbations from various types of variation affect the stability of the solution to the problem of signal separation.

For purposes of further discussion, we will refer to matrices of parameter intervals varying from the initial state $\mathbf{H}(\omega, \mathbf{l})$ to the degenerated (singular) state $\mathbf{H}(\omega, \mathbf{l})$ as singular-interval matrices and designate them $\Delta \mathbf{H}(\omega, \mathbf{l})$.

Among the different types of variations, we will consider those most often encountered in engineering practice-absolute, relative, and critical variations, which simulate related real perturbations [7]. Critical variations are variations that cause the initial model $(5,6)$ to become degenerated at a minimal spectral variation norm of $\|\delta \mathbf{H}(\omega, \mathbf{l})\|_{2}$. Relative variations, as the name suggests, have values proportional to those of matrix elements. Absolute variations can be of any value unconnected with the value of the current matrix element. Thus, this paper aims to determine singular intervals for parameters $\Delta \mathbf{H}_{a b s}(\omega, \mathbf{l}), \Delta \mathbf{h}_{a b s}(g, \mathbf{l})$, $\Delta \mathbf{H}_{r e l}(\omega, \mathbf{l}), \Delta \mathbf{h}_{r e l}(g, \mathbf{l}), \Delta \mathbf{H}_{c r i t}(\omega, \mathbf{l})$, and $\Delta \mathbf{h}_{c r i t}(g, \mathbf{l})$ for the variations above. In the introduced matrices of singular parameter intervals, $m n$th elements $\Delta H_{m n}$ indicate changes in parameters of $m n$th elements of the initial matrix $\mathbf{H}(\omega, \mathbf{l})$.

In particular, if model channels are frequency-independent and parameter vector $\mathbf{l}$ independent, the designations of singularinterval matrices omit arguments $(\omega),(g)$ and $(\mathbf{l})$; for instance, $\Delta \mathbf{H}_{a b s}, \Delta \mathbf{h}_{a b s}$.

Thus, the singular intervals obtained from calculations reflect those absolute, relative, and critical perturbations of the signal formation model's parameters that cause the model to be unstable. 


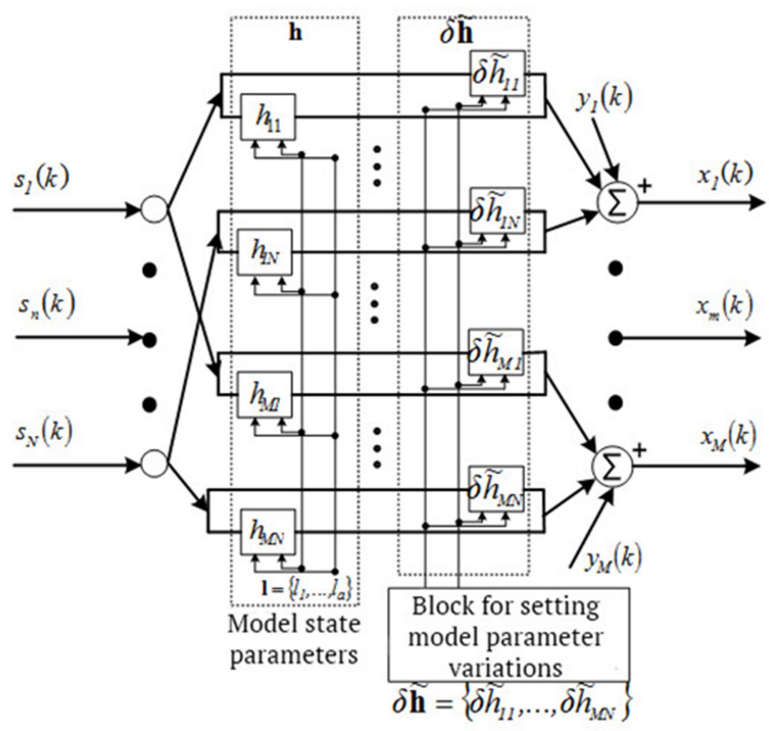

Fig. 1. Schematic of the signal formation model with the capability to set variations for channel parameters to analyze the stability of signal separation.

\subsection{Analyzing and Verifying the Stability of Signal Separation by Determining Singular Parameter Intervals for the Signal Formation Model}

For purposes of determining singular parameter intervals for different variations, a generalized algorithm has been developed $[7,10]$, in which three stages can be distinguished: determining the singular direction of parameter variation; determining a singular matrix; and determining a singular-interval matrix.

Singular directions for absolute, relative, and critical variations are determined by direction matrices whose analytic expressions, obtained in references [7,10], are given in table 1 .

Direction matrices can be calculated both on the basis of singular value decomposition (SVD) and on the basis of the inverse matrix. Determining singular directions with proposed matrices $\mathbf{Z}$ has lower computational complexity compared with known algorithms.

\begin{tabular}{|c|c|c|c|}
\hline \multirow{2}{*}{$\begin{array}{l}\text { Calculation } \\
\text { method }\end{array}$} & \multicolumn{3}{|c|}{ Type of variation } \\
\hline & Critical & Absolute & Relative \\
\hline \multirow{2}{*}{$\begin{array}{l}\text { Based on } \\
\text { inverse } \\
\text { matrix }\end{array}$} & $\mathbf{H}^{-*}$ & $\mathbf{A} \mid \otimes \operatorname{sign}\left(\mathbf{H}^{-*}\right)$ & $|\mathbf{H}| \otimes \operatorname{sign}\left(\mathbf{H}^{-*}\right)$ \\
\hline & $\left\|\mathbf{H}^{-1}\right\|_{2}$ & $\| \mathbf{A} \mid \otimes \operatorname{sign}\left(\mathbf{H}^{-*)} \|_{1}\right.$ & $\left\|\mathbf{H} \mid \otimes \operatorname{sign}\left(\mathbf{H}^{-*}\right)\right\|$ \\
\hline \multirow{2}{*}{$\begin{array}{l}\text { Based on } \\
\text { SVD }\end{array}$} & \multirow{2}{*}{$-u_{N} v_{N}^{*}$} & $|\mathbf{A}| \otimes \operatorname{sign}\left(u_{N} v_{N}^{*}\right)$ & $|\mathbf{H}| \otimes \operatorname{sign}\left(u_{N} v_{N}^{*}\right)$ \\
\hline & & || $\mathbf{A} \mid \otimes \operatorname{sign}\left(u_{N} v_{N}^{*}\right)$ & $|\mathbf{H}| \otimes \operatorname{sign}\left(u_{N} v_{N}^{*}\right) \mid$ \\
\hline
\end{tabular}

The proposed matrices $\mathbf{Z}$ can be used to determine singular parameter intervals not only for real elements (as in known algorithms) but also for complex (frequency-dependent) elements of mixing matrix $\mathbf{H}(\omega)$.

Table 1 refers to: $|\mathbf{A}|$, a matrix composed of element modules of matrix $\mathbf{A}$ and determining absolute parameter variations; $\operatorname{sign}(\mathbf{A})$, matrix operation whose elements are calculated as $\operatorname{sign}\left(A_{m n}\right)=A_{m n} /\left|A_{m n}\right| ; \otimes$, element-by-element multiplication of matrices $\mathbf{C}=\mathbf{A} \otimes \mathbf{B}$, where $C_{m n}=A_{m n} \cdot B_{m n} ; v_{n}$ and $u_{n}$, right and left singular vectors of singular value decomposition $\mathbf{H}=\mathbf{U} \boldsymbol{\Sigma} \mathbf{V}^{*}=\sum_{n=1}^{N} \sigma_{n} u_{n} v_{n}^{*} ;\|\|_{1}$, the maximum column sum matrix norm.

It is proposed that singular matrix $\mathbf{H}$ be calculated by finding the roots of the equation $f\left(\mathbf{H}_{j}+\delta h \cdot \mathbf{Z}\right)=\operatorname{det}\left(\mathbf{H}_{j}+\delta h \cdot \mathbf{Z}\right)=0$ under restrictions caused by parameter variations.

The numerical algorithm (table 2) for determining singular matrices $\mathbf{H}$ for absolute, relative, and critical variations is based on the Newton method, in which, unlike in the classical method, derivative $f_{Z_{j}}^{\prime}\left(\mathbf{H}_{j}\right)$ is calculated on the basis of the matrix of directions $\mathbf{Z}$ and refined in each step. This improves accuracy and simplifies computation compared with known algorithms [10].

At the third stage, singular-interval matrix $\Delta \mathbf{H}$ is calculated as follows: $\Delta \mathbf{H}=\mathbf{H}-\mathbf{H}$. In the proposed algorithm, function $f\left(\mathbf{H}_{j}+\delta h \cdot \mathbf{Z}\right)$ must satisfy the conditions of convergence theorems from the Newton method, including the Lipschitz condition. 


\begin{tabular}{|c|c|c|}
\hline Step & Action & Note \\
\hline 1 & $\begin{array}{l}\text { Parameter } \gamma>0 \text { is set, and it determines error, increment value } \delta h \text {, } \\
\text { and initial iteration value } j=1\end{array}$ & $\begin{array}{l}\text { Initialization } \\
\text { takes place }\end{array}$ \\
\hline 2 & $\begin{array}{l}\mathbf{Z}_{j} \text { is determined according to type of parameter variation } \\
\text { (table 1) for matrix } \mathbf{H}_{j}\end{array}$ & $\begin{array}{l}\text { Direction matrix } \\
\text { is calculated }\end{array}$ \\
\hline 3 & $f_{\mathbf{Z}_{j}}^{\prime}\left(\mathbf{H}_{j}\right)=\frac{d f\left(\mathbf{H}_{j}\right)}{d \mathbf{Z}_{j}}=\frac{f\left(\mathbf{H}_{j}+\delta h \cdot \mathbf{Z}_{j}\right)-f\left(\mathbf{H}_{j}\right)}{\delta h}$ & $\begin{array}{l}\text { Derivative is calculated for } \\
\text { direction } \mathbf{Z}_{j}\end{array}$ \\
\hline & $\delta \mathbf{H}_{j}=-\frac{\mathbf{Z}_{j} \cdot f\left(\mathbf{H}_{j}\right)}{f_{\mathbf{z}_{j}}^{\prime}\left(\mathbf{H}_{j}\right)}$ & $\begin{array}{l}\text { Matrix for singular variations of } \\
\text { parameters } \delta \mathbf{H}_{j} \text { is calculated }\end{array}$ \\
\hline 5 & $\mathbf{H}_{j+1}=\mathbf{H}_{j}+\delta \mathbf{H}_{j}$ & $\begin{array}{l}\text { New approximation } \\
\text { is calculated }\end{array}$ \\
\hline 6 & $\begin{array}{l}\text { If }\left|f\left(\mathbf{H}_{j+1}\right)-f\left(\mathbf{H}_{j}\right)\right|<\gamma, \text { then algorithm ends }\left(\mathbf{H}=\mathbf{H}_{j}\right) \text {; else, } \\
j=j+1, \text { go to step } 2\end{array}$ & $\begin{array}{l}\text { Algorithm completion } \\
\text { is verified }\end{array}$ \\
\hline
\end{tabular}

The method for calculating singular intervals provided the basis for algorithms for verifying the stability of signal separation. Table 3 outlines an example algorithm [10].

Table 3. Algorithm for verifying the stability of the solution to the inverse problem of signal separation.

Step Action Note

\begin{tabular}{|c|c|c|}
\hline 1 & Frequency index $g=0$ is set for spectral matrix $\mathbf{H}\left(\omega_{g}, \mathbf{l}\right)$ & Initialization \\
\hline 2 & $\begin{array}{l}\text { Singular-interval matrix } \Delta \mathbf{H}\left(\omega_{g}, l\right)=\mathbf{H}\left(\omega_{g}, l\right)-\mathbf{H}\left(\omega_{g}, l\right) \text { is } \\
\text { calculated }\end{array}$ & For frequency $\omega_{\mathrm{g}}$ \\
\hline 3 & Threshold matrix $\mathbf{H}_{t}\left(\omega_{g}, l\right)$ is determined & For frequency $\omega_{\mathrm{g}}$ \\
\hline 4 & $\begin{array}{l}\text { Matrices are determined for intervals of parameters of } \\
\text { stable }\left(\Delta \mathbf{H}_{R}\left(\omega_{g}, \mathbf{l}\right)=\mathbf{H}\left(\omega_{g}, \mathbf{l}\right)-\mathbf{H}_{t}\left(\omega_{g}, \mathbf{l}\right)\right) \text { and } \\
\text { unstable }\left(\Delta \mathbf{H}_{S}\left(\omega_{g}, \mathbf{l}\right)=\mathbf{H}\left(\omega_{g}, \mathbf{l}\right)-\mathbf{H}_{\Pi}\left(\omega_{g}, \mathbf{l}\right)\right) \text { separation }\end{array}$ & For frequency $\omega_{\mathrm{g}}$ \\
\hline 5 & $\begin{array}{l}\text { If }\left|\Delta \mathbf{H}_{\max }\left(\omega_{g}, \mathbf{l}\right)\right| \leq\left|\Delta \mathbf{H}_{R}\left(\omega_{g}, \mathbf{l}\right)\right| \text {, solution is stable; otherwise message appears } \\
\text { stating that stable signal separation for frequency of } \omega_{\mathrm{g}} \text { is impossible }\end{array}$ & $\begin{array}{l}\text { Conditions of } \\
\text { separation stability } \\
\text { at frequency of } \omega_{\mathrm{g}} \\
\text { are verified }\end{array}$ \\
\hline 6 & $\begin{array}{l}\text { If }\left|\Delta \mathbf{H}_{p o t}\left(\omega_{g}, \mathbf{l}\right)\right| \leq\left|\Delta \mathbf{H}_{R}\left(\omega_{g}, \mathbf{l}\right)\right| \text {, solution is stable; otherwise message appears } \\
\text { stating that stable signal separation for frequency of } \omega_{\mathrm{g}} \text { is not guaranteed }\end{array}$ & $\begin{array}{l}\text { Conditions of stable } \\
\text { separation } \\
\text { at frequency of } \\
\omega_{\mathrm{g}} \text { are verified }\end{array}$ \\
\hline 7 & $\begin{array}{l}g=g+1 . \text { If } g>G-1 \text {, algorithm ends, and final message on stability verification } \\
\text { appears; else, step } 2\end{array}$ & $\begin{array}{l}\text { Transition to } \\
\text { next spectral matrix }\end{array}$ \\
\hline
\end{tabular}

The matrix $\Delta \mathbf{H}_{\max }\left(\omega_{g}, \mathbf{l}\right)$ added in step 5 for maximal allowable variation intervals is defined from theoretical and practical information about the object being modeled. Matrix inequalities of $|\mathbf{A}| \leq|\mathbf{B}|$ type should be understood as systems of componentwise inequalities $\left|A_{m n}\right| \leq\left|B_{m n}\right|$.

Threshold matrix $\mathbf{H}_{t}\left(\omega_{g}, \mathbf{l}\right)$, determined in step 3 of the algorithm, is mixing matrix $\mathbf{H}_{j}\left(\omega_{g}, \mathbf{l}\right)$, for which cond $\mathbf{H}_{j}\left(\omega_{g}, \mathbf{l}\right)$ exceeds a given threshold value of cond $_{\mathrm{t}}$. To determine matrix $\mathbf{H}_{t}\left(\omega_{g}, \mathbf{l}\right)$, mixing matrix $\mathbf{H}_{j}\left(\omega_{g}, \mathbf{l}\right)$ is changed in accordance with the expression $\mathbf{H}_{j}\left(\omega_{g}, \mathbf{l}\right)=\mathbf{H}\left(\omega_{g}, \mathbf{l}\right)+j \times \delta \mathbf{H}\left(\omega_{g}, \mathbf{l}\right)$, and in each step $j=1, \ldots, J$ its cond $\mathbf{H}_{j}\left(\omega_{g}, \mathbf{l}\right)$ is compared with threshold value cond $_{t}$.

The calculated matrix $\mathbf{H}_{t}\left(\omega_{g}, \mathbf{l}\right)$ of threshold values serves as the basis for determining the matrix of parameter intervals for stable separation $\Delta \mathbf{H}_{R}\left(\omega_{g}, l\right)=\mathbf{H}\left(\omega_{g}, \mathbf{l}\right)-\mathbf{H}_{t}\left(\omega_{g}, \mathbf{l}\right)$ and the matrix of parameter intervals for unstable separation $\Delta \mathbf{H}_{S}\left(\omega_{g}, \mathbf{l}\right)=\mathbf{H}\left(\omega_{g}, \mathbf{l}\right)-\mathbf{H}_{t}\left(\omega_{g}, \mathbf{l}\right)$.

\section{Results and Discussion}

Figure 2 shows the relationship between relative error $\xi_{\Delta H}$ in determining singular intervals $\Delta \mathbf{H}$ and the reduced error of the parameters of mixing matrix $\mathbf{H}$ (determined by the number of binary digits of the ADC) and its values cond ( $\mathbf{H})$ for critical parameter variations. Figure 3 shows results testing algorithms for verifying the stability separation of signals. 


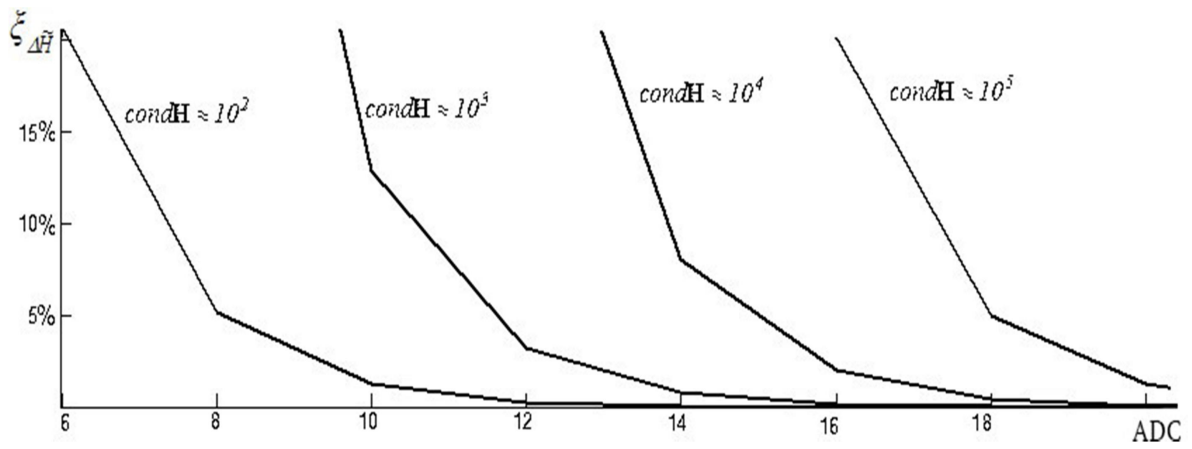

Fig. 2. The relationship between relative error $\xi_{\Delta H}$ in determining singular intervals and the reduced error of matrix $\mathbf{H}$ parameters (determined by the
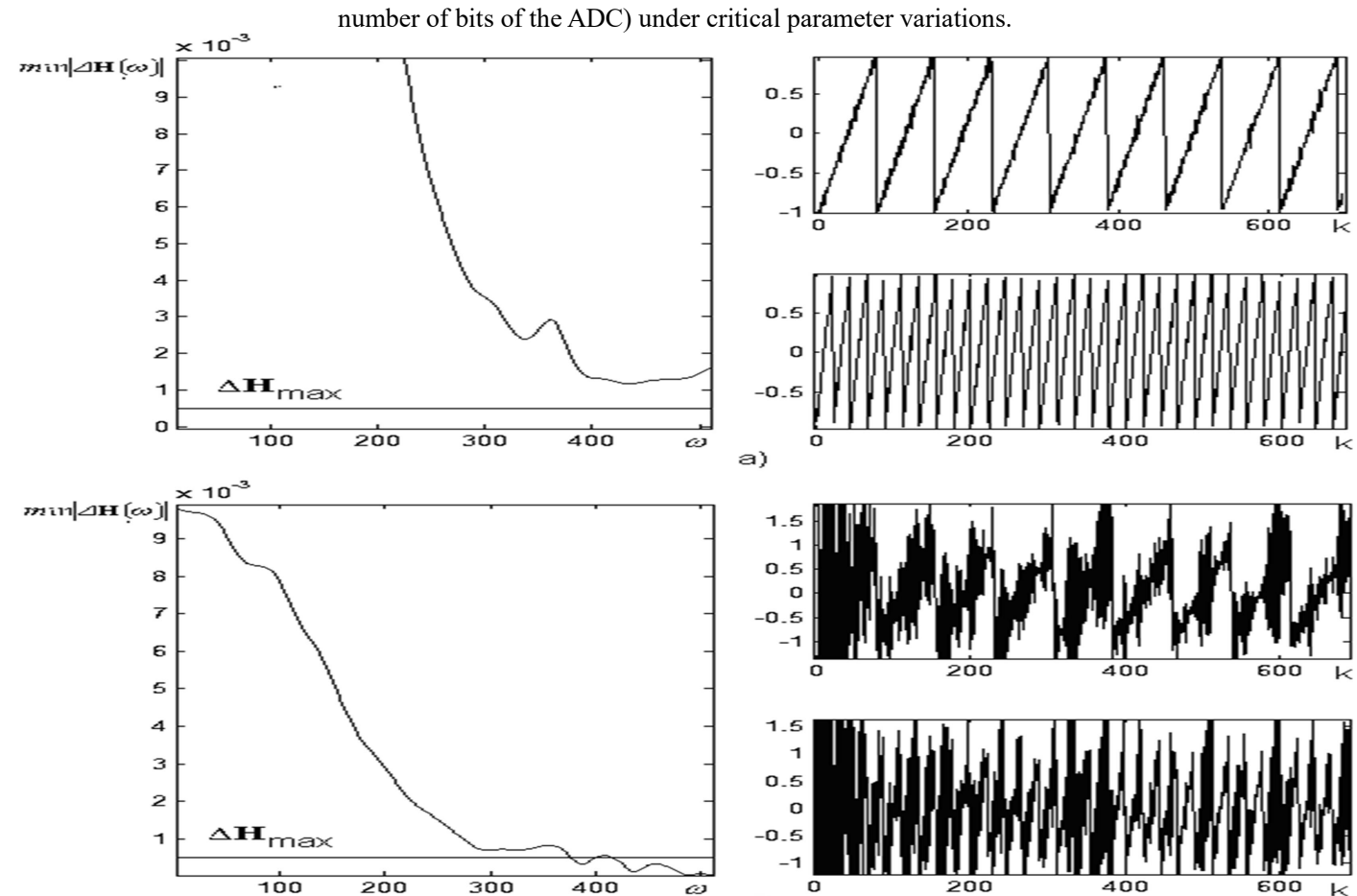

a)
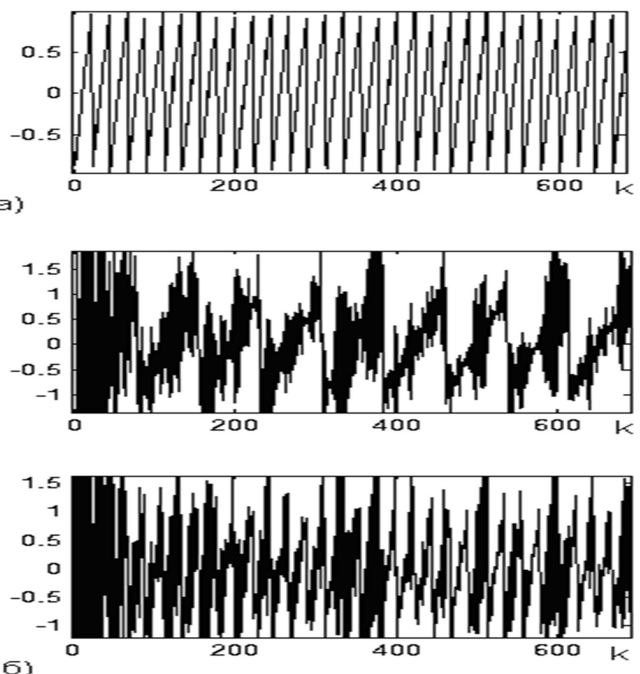

Fig. 3. Results testing algorithms for verifying the stability: illustrate stable (a) and unstable (b) separation of signals

The proposed algorithms have been incorporated in a software system for modeling signal separation and restoration. Figure 4 shows examples of modeling and investigating the stability of signal separation for test signals and signals in an automatic cab signaling system, which transmits, via the track, traffic-light coding signals to train cab [11].

The automatic cab signaling system, which is used for train safety, operates under the influence of various interference sources. Suppressing that interference is important for the reliable operation of the system. Sometimes for interference sources to be suppressed, interference signals need to be extracted in order to determine their physical nature; that is, to identify interference sources [11]. This needs to be done as part of monitoring the condition of track circuits and automatic cab signaling systems.

Figures 4-a(1) and 4-a(2) show initial triangular test signals and their mixtures, and figures 4-a(3) и 4-a(4) are examples of a unstable and an stable solution to the problem of separating test signals. The Information window indicates that the separation is unstable: singular intervals at a frequency of $400 \mathrm{~Hz}$ are close to zero, and the condition number is on a sharp increase.

Figures 4-b(1) and 4-b(2) show examples of the automatic cab signaling system's amplitude-modulated signals under the influence of interference: fluctuation noise from traction current, a $50 \mathrm{~Hz}$ harmonic interference from the power line, and a lowfrequency interference of $4 \mathrm{~Hz}$ due to intake coils' wobbling in relation to the track. Figures 4-b(3) and 4-b(4) illustrate unstable and stable separation of signals in the system and the above interference. Whether the solution is stable or unstable is displayed in the Information window.

This results confirms the possibility of using the developed algorithms in engineering applications.

\section{Conclusion}

The key results of our investigation are as follows:

We proposed an algorithm for modeling signal separation that makes it possible to study the stability of solutions to the problem of signal separation under stability-crucial parameter variations (perturbations) controlled in a signal formation model.

We also developed algorithms for analyzing and verifying stability by determining singular intervals for parameters of the signal formation model for various parameter variations. 


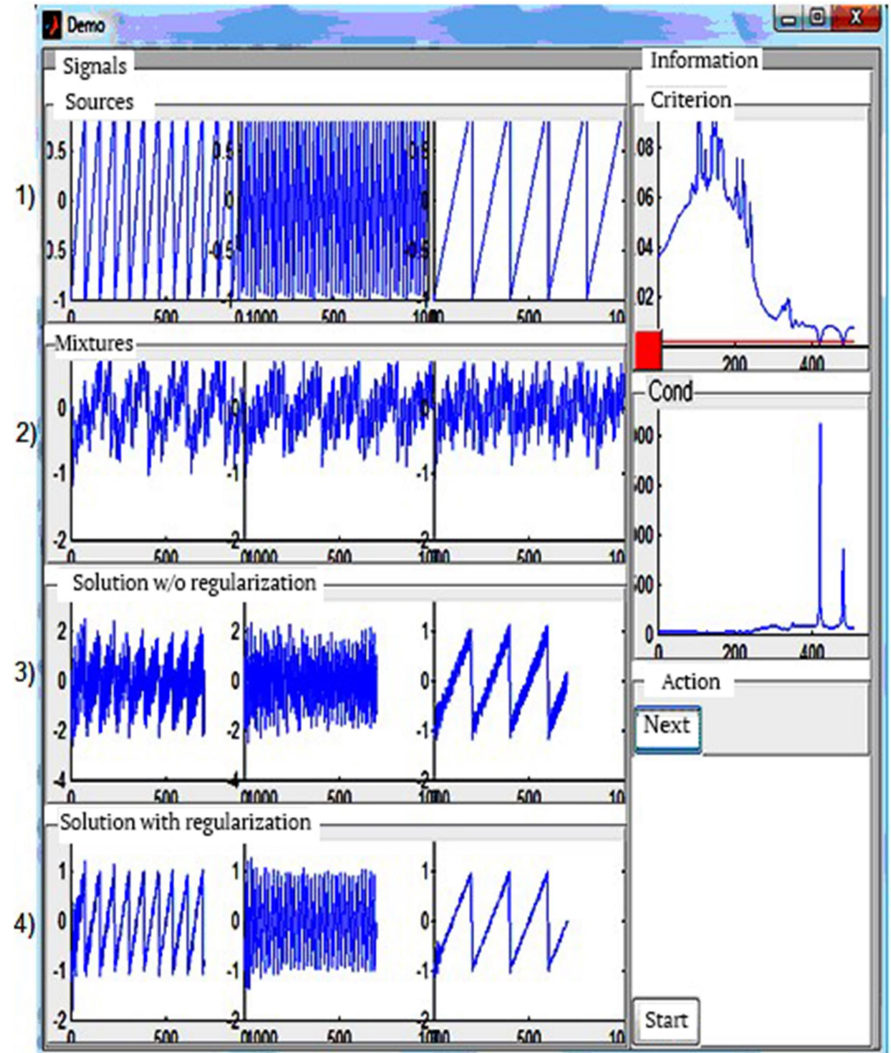

a)

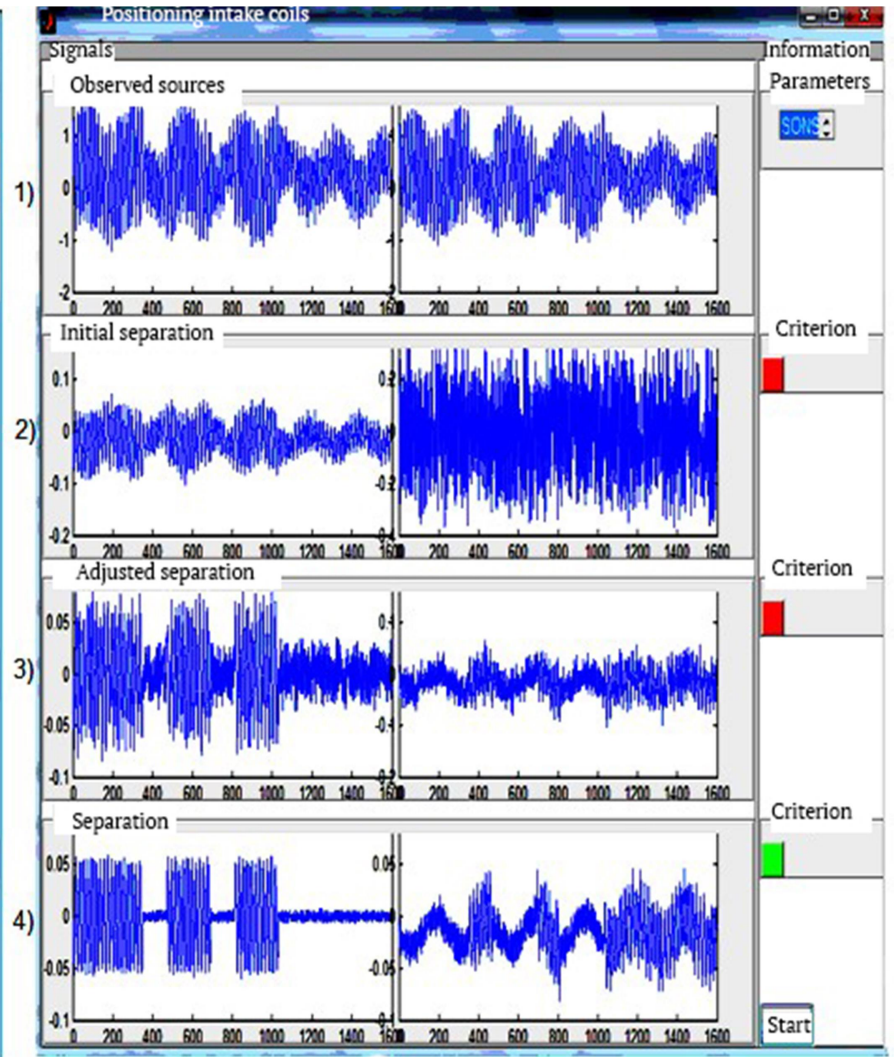

b)

Fig.4. Results of analyzing the stability of the solution to the problem of signal separation: a) test signals; b) measured signals in the automatic cab signaling system.

\section{References}

[1] Bakushinskiy AB, Goncharskiy AV. Ill-Posed Problems: Numerical Methods and Applications. Moscow: Moscow State University Press, 1989 ; 199 p. (in Russian)

[2] Petrov Yu.P, Sizikov VS. Well-Posed, Ill-Posed, and Intermediate Problems with Applications: A Textbook for Institutes of Higher Education. Saint Petersburg: "Politekhnika" Publisher, 2003; 261 p. (in Russian)

[3] Digital Signal and Image Processing in Radiophysical Applications. Ed. Kravchenko VF. Moscow: "Fizmatlit” Publisher, 2007; 544 p. (in Russian)

[4] Tyrtyshnikov YeYe. Matrix Analysis and Linear Algebra. Moscow: "Fizmatlit" Publisher, 2007; 480 p. (in Russian)

[5] Demmel J. Applied Numerical Linear Algebra. Theory and applications. Moscow: "Mir" Publisher, 2001 ; 430 p. (in Russian)

[6] Petrov Yu P. Obtaining Reliable Solutions to Equation Systems. Saint Petersburg: Izdatelstvo "BKhV-Peterburg" Publisher, 2009 ; 176 p. (in Russian)

[7] Zasov VA. Algorithms and Computational Devices for Separating and Restoring Signals in Multivariable Dynamic Systems: A Monograph. Samara: Samara State Transport University Press, 2013; 233 p. (in Russian)

[8] Cichocki A, Amari Sh. Adaptive Blind Signal and Image Processing: Learning algorithms and applications. John Wiley \& Sons Ltd, $2002 ; 555$ p.

[9] Windrow B, Stearns S. Adaptive Signal Processing. Moscow: "Radio i svyaz" Publisher, 1989; 440 p. (in Russian)

[10] Zasov VA, Nikonorov YeN. Algorithms for Verifying the Stability of a Solution to the Problem of Separating Signal Sources under Conditions of Prior Uncertainty. Published in Hardware and Software Means for Management, Control, and Measurement Systems: Proceedings of a Conference with Russian and International Attendance. Moscow: Russian Academy of Sciences, Trapeznikov Institute of Control Sciences Press, $2010 ; 482-491$. (in Russian)

[11] Zasov VA, Nikonorov YeN, Tarabardin MA. Identifying Input Signals in Problems of Controlling and Diagnosing Dynamic Objects. Proceedings of the IV International Conference on Control Problems. Moscow: Russian Academy of Sciences, Trapeznikov Institute of Control Sciences Press, 2009; 1478-1486. (in Russian) 